\title{
Chondral Defect Repair with Particulated Juvenile Cartilage Allograft
}

\author{
Jack Farr' and Jian Q. Yao
}

\begin{abstract}
Objective: This case study of 4 patients followed for at least 2 years was conducted to evaluate a cartilage repair procedure that involves transplanting particulated juvenile allograft cartilage. Design: A multicenter, prospective, single-arm, 25-subject case study was designed to evaluate clinical outcomes such as IKDC and KOOS scores as well as the extent and quality of repair with MRI. In addition, there is an option for the transplants to be biopsied at various time points after implantation (up to 5 years). Currently, 25 patients with I or 2 chondral lesions on the femoral condyles and trochlea have been enrolled and treated in the prospective study. Results: The first 4 patients have completed an evaluation at 24 months postoperative follow-up. Improvements in clinical outcomes over the preoperative baseline data have been observed. Conclusions: The present report describes, for the first time, clinical intermediate-term results of a novel cartilage repair procedure that involves transplanting particulated juvenile cartilage tissue allograft into prepared cartilage lesions of the femoral condyles and/or trochlea. Clinical outcome data of 4 patients who have reached the 24-month postimplantation milestone indicate early positive outcomes and suggest that this technique is capable of improving clinical symptoms. MRI data suggest that defect filling is possible and persists to at least 2 years. Continued clinical evaluation of this technique is needed with extended follow-up of all 25 patients in the series.
\end{abstract}

\section{Keywords}

articular cartilage, particulated, juvenile, allograft, clinical

\section{Introduction}

Articular cartilage lesions of the knee are common. In the United States, the surgical treatment options for symptomatic patients include debridement/lavage, ${ }^{1}$ marrow stimulation, ${ }^{2}$ osteochondral autograft transplantation, ${ }^{3}$ fresh osteochondral allograft transplantation, ${ }^{4}$ and autologous chondrocyte implantation. ${ }^{5,6}$ More recently, an alternative technique using transplantation of particulated juvenile cartilage allograft has become available. ${ }^{7}$

In the past, allograft transplants were limited to osteochondral grafts because graft incorporation to host tissue was only possible at the bone level; the desired live articular cartilage transplant needed to remain attached to its underlying subchondral bone. It has been generally accepted that cartilage grafts without the subchondral bone attached would not be incorporated to either host cartilage (failure of lateral integration) or host subchondral bone (failure of basilar integration). ${ }^{8}$ In fact, both autologous and allogeneic osteochondral grafts do not heal at the lateral margins (i.e., no donor cartilage/host cartilage integration is seen); only the basilar bone portion heals, which retains the overlying cartilage cap in its proper position. The host "heals" to the transplant through creeping substitution of the patient's bone into the transplanted bone. In some cases, however, it has been suggested that host immunological responses to the bony portion of the osteochondral allograft led to possible nonincorporation and necrosis of the graft bone. Bugbee ${ }^{9}$ reported that the greater the amount of transplant material used, the more patients become "antibody positive" and the higher the failure rate in this subset of patients. Historically, the majority of failures reported in early clinical series of osteochondral transplants were related to failure of the dead bone (e.g., thicker segments of bone failed with collapse similar to avascular necrosis). Thus, the amount of bone transplanted has been progressively decreased to the point that the technique is now termed osteochondral shell transplantation with cartilage-bone composite thicknesses of only 6 to $8 \mathrm{~mm}$. The concept that cartilage could be transplanted without its underlying bony component and heal would have been considered illconceived by most cartilage surgeons even a few years ago.

\footnotetext{
'Ortholndy, Cartilage Restoration Center of Indiana I260 Innovation Parkway Suite 100, Greenwood, IN, 46I43, USA

${ }^{2}$ Research and Development, Zimmer Orthobiologics, Inc., 930I

Amberglen Blvd., Suite 100, Austin, TX 78729, USA

Corresponding Author:

Jack Farr, 1260 Innovation Parkway Suite I00, Greenwood, IN, 46I43, USA Email: indyknee@hotmail.com
} 
Notwithstanding this widely held skepticism regarding the clinical efficacy of chondral grafts, the development of a particulated juvenile cartilage allograft and methods of surgically implanting the same were undertaken via a series of in vitro and in vivo animal studies. The preliminary demonstration of human clinical efficacy reported here is consistent with basic science research findings from these preclinical studies showing that new extracellular matrix can be formed between 2 adjacent juvenile cartilage cubes in an explant culture study and that particulated juvenile cartilage xenografts healed chondral defects on trochlear areas of horse knee joints. In addition, the present results expand and improve on prior efforts to treat cartilage defects with either particulated chondral or osteochondral tissue from mature donors. For example, Stone et al. ${ }^{10}$ implanted a paste of autologous osteochondral tissue into cartilage defects concomitantly treated with microfracture. Although Stone et al. reported good clinical results, animal studies showed that the combination of bone and cartilage in a paste formed bone and cartilage, whereas cartilage pieces alone formed cartilage. ${ }^{10}$ Similarly, Albrecht et al. ${ }^{11}$ showed that cartilage allograft transplantation led to cartilage (and bone) defect healing in the rabbit when the investigators implanted small pieces of mature articular cartilage in a full-thickness defect that also had the bone bed drilled. In that study, it appeared that chondrocytes within the cartilage pieces migrated out of the cartilage matrix, multiplied, and formed new extracellular matrix (i.e., hyaline-like cartilage tissue) and integrated with surrounding host cartilage. Further animal studies in the SCID mouse, goat, and horse models showed that particulated adult cartilage grafts can remodel to form a continuous volume of repair tissue. ${ }^{12,13}$

Observations regarding the age-related differences in spontaneous healing and histological and biochemical characteristics of cartilage may suggest some potential mechanisms associated with the clinical performance of particulated juvenile cartilage allografts. For example, injured immature cartilage has been shown to heal spontaneously, whereas injured adult cartilage cannot. ${ }^{14,15}$ In addition, immature articular cartilage tissue has a significantly higher cell density than mature articular cartilage. ${ }^{16}$ Moreover, compared with mature chondrocytes, immature chondrocytes are capable of producing greater amounts of extracellular matrix as measured by the rate of production of sulfated glycosaminoglycans ${ }^{7}$ and greater levels of messenger RNA for Type II and Type IX collagen. ${ }^{18}$

The present article details the results to date of an institutional review board (IRB)-approved prospective study of particulated juvenile cartilage allograft (DeNovo NT Natural Tissue Graft) (Zimmer, Inc., Warsaw, IN, and ISTO Technologies, Inc., St Louis, MO) transplanted in human knees, with a subset ( 4 of 25 ) of patients currently at 24 months after surgery. The objective of this case study was to evaluate the initial clinical performance of this cartilage repair technique. It should be noted that in addition to 25 cases in this series, as of March 2011, there were more than 2,200 DeNovo NT implantation procedures performed. Furthermore, this is a minimally manipulated human tissue allograft product regulated in the United States as a 361 $\mathrm{HCT} / \mathrm{P}$ product, in the same manner as fresh osteochondral allografts, meniscus allograft, bone-tendon-bone allograft, and cancellous allograft bone chips.

\section{Methods}

DeNovo NT graft was prepared by carefully removing live cartilage tissue from fresh cadaveric juvenile femoral condyles (up to 13 years old), particulating them manually into small cubes of approximately $1 \mathrm{~cm}^{3}$, and packaging the tissue pieces in sealed blister packs containing a nutrient medium.

The prospective case series investigation was IRB approved at each of the 3 study sites. Symptomatic patients between the age of 18 and 55 with 1 or 2 contained focal chondral lesions involving the medial femoral condyle, the lateral femoral condyle, and/or the trochlea were eligible to participate. Study participants needed to have near-normal alignment (varus or valgus $5^{\circ}$ or less from neutral mechanical axis), functional menisci, and a stable knee. Clinically qualified patients were consented for participation in the study, and final satisfaction of the inclusion/exclusion criteria was confirmed at arthroscopy before patients were treated with DeNovo NT graft.

The key inclusion and exclusion criteria are listed in Tables 1 and 2.

Preoperatively and sequentially postoperatively, enrolled patients completed patient-reported outcome forms consisting of the Knee injury and Osteoarthritis Outcome Score (KOOS), International Knee Documentation Committee (IKDC) Subjective Knee Evaluation score, and visual analog scale (VAS) pain score. KOOS was not collected at 6 and 18 months postimplantation. The preoperative radiographs and MRIs were compared with those at different follow-up time points ( 6 months, 1 year, and then yearly for 5 years). Histology evaluation was planned for any elective biopsies to be obtained at 24 months postoperative.

\section{Surgical Technique}

After confirmatory arthroscopy, a limited medial or lateral arthrotomy was perform to fully visualize the lesions (Fig. 1A). The defect was outlined with a scalpel to create a shoulder (vertical peripheral wall) of normal or nearly normal host articular cartilage. The cartilage within the outlined area was removed carefully with a curette to the vertical wall of the host cartilage shoulder and the base of the defect (Fig. 1B). The base was cleared of all cartilage tissue. The calcified layer was removed very carefully as much as practically 
Table I. Inclusion Criteria

I. Subjects who have signed voluntarily the IRB-approved informed consent

2. Male or female subjects between the ages of 18 and 55 years

3. Subjects with pretreatment arthroscopic confirmation of I or 2 contained lesions equal to an ICRS Grade 3a, 3b, 3c, 3d of the femoral condyle or trochlear groove and osteochondritis dissecans lesions (Grade 4a) with healed bone base, which is nonsclerotic, and no loss of bone $>6 \mathrm{~mm}$ measured from the surrounding subchondral plate

4. Subjects with a lesion(s) with a postdebridement area of $\geq 1 \mathrm{~cm}^{2}$ and $\leq 5 \mathrm{~cm}^{2}$

5. Subjects with stable posterior cruciate ligament, lateral collateral ligament, and medial collateral ligament in the affected knee and an anterior cruciate ligament that is stable or can be stabilized in a concomitant procedure

6. Subjects with intact menisci (or stable menisci after a concomitant partial meniscectomy procedure) in the ipsilateral knee compartment

Table 2. Exclusion Criteria

I. Subjects with clinical and/or radiographic disease diagnosis of the indexed affected joint, including the following:

- Osteoarthritis or avascular necrosis

- Rheumatoid arthritis or history of septic or reactive arthritis

- Gout or a history of gout or pseudogout in the affected knee

- Osteochondritis dissecans of the knee with significant bone loss (> $6 \mathrm{~mm}$ deep from the subchondral plate)

- Associated damage to the underlying subchondral bone requiring an osteochondral graft

2. Subjects with HIV or other immunodeficient states who are undergoing immunosuppressant therapies, or subjects with significant illness (metastasis of any type) that decreases the probability of survival to the 2-year end point

3. Subjects with body mass index $>35$ (body mass index $=\mathrm{kg} / \mathrm{m}^{2}$ )

4. Subjects with bipolar articular cartilage involvement or kissing lesions of the ipsilateral compartment, described as tibial or patellar lesions in the same compartment with greater than ICRS Grade 2 chondrosis

5. Subjects with active joint infection

6. Subjects with prior total meniscectomy of either knee

7. Subjects having radiographically $>5^{\circ}$ of malalignment as measured from the hip, knee, and ankle mechanical axis

8. Subjects having received, within the past 3 months, intra-articular hyaluronic acid therapy or cortisone injections in the index knee

9. Subjects having undergone a prior realignment surgery in the affected knee within the past 6 months

10. Subjects having failed microfracture treatment performed $<12$ months before baseline

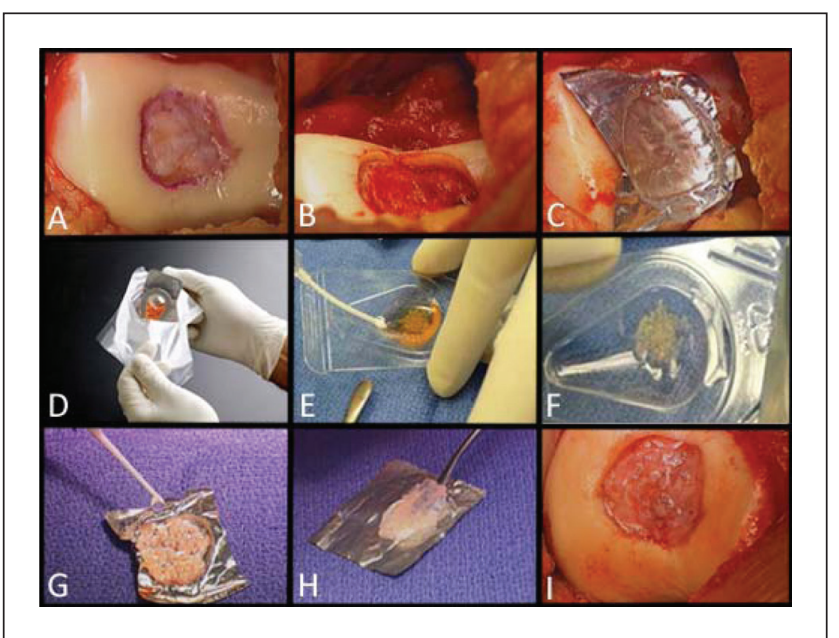

Figure I. Surgical technique.

possible without entering into the subchondral bone. No intentional marrow stimulation procedure was performed. Incidental bleeding, however, did occur sometimes and was stopped in the similar manner as in an autologous chondrocyte implantation (ACI) procedure with epinephrine-soaked cottonoids and/or fibrin glue (with the tourniquet deflated). After we measured the defect dimensions and recorded the visual findings with photographs, a thin aluminum sterile foil was pressed into the defect to create a 3-dimensional mold, as a complete replica of the defect (Fig. 1C). Once formed, the foil mold was removed from the defect and placed on the back table of the operating room. The measured defect dimensions were used to calculate the defect surface area. One package of DeNovo NT graft was used for each $2.5 \mathrm{~cm}^{2}$ of defect area. Larger defects required proportionally more packages of DeNovo NT graft. The DeNovo NT graft, in a nutrient medium, was shipped in a temperature-controlled package (Fig. 1D). The nutrient medium was aspirated (Fig. 1E and F), and the particulated cartilage pieces were transferred to the foil mold and distributed approximately $1 \mathrm{~mm}$ apart (potentially less separation depending on the ratio between the implanted tissue volume and the surface area of the defect) (Fig. 1G). Fibrin glue was then added to the cartilage pieces and allowed to 
Table 3. Age, Gender, and Body Mass Index of the 4 Patients

\begin{tabular}{lclc}
\hline Subject & Age, Years & Gender & Body Mass Index, lb/in \\
\hline $\mathrm{I}$ & 38 & Male & 34 \\
2 & 46 & Female & 20 \\
3 & 39 & Male & 28 \\
4 & 49 & Male & 26 \\
Mean (SD) & $43(5.4)$ & & $27(5.8)$ \\
Min, Max, N & $38,49,4$ & & $20,34,4$ \\
\hline
\end{tabular}

cure (Fig. 1G). At that point, the cartilage tissue construct was gently separated and then lifted from the foil in 1 piece (Fig. 1H). Fresh fibrin glue was applied at the base of the patient's cartilage lesion, the fibrin glue/cartilage construct was pressed into the defect, and the glue was allowed to cure for approximately 10 minutes (Fig. 1I). The fibrin glue-cartilage tissue construct was recessed relative to the surrounding cartilage shoulders (average 2-3 mm), which would minimize the potential for shear or direct compressive load. The patients underwent a standard postoperative protocol that mimicked the Steadman microfracture proto$\mathrm{col}^{19}$; this type of protocol has been used for other pilot studies such as DeNovo ET (Engineered Tissue; ISTO, St. Louis, MO, and Zimmer, Warsaw, IN) and CAIS (DePuy/ Mitek, Raynham, MA) as reported on www.clinicaltrials.gov.

\section{Results}

\section{Study Subjects}

To date, 25 patients have been enrolled at 3 study sites, 4 of whom have been followed for 24 months. In this report, we present the clinical data on these 4 patients only (Table 3 ). Of these 4 patients, 3 had nontraumatic cartilage lesions and 1 had a traumatic cartilage injury. Table 4 lists the prior surgeries (if any) that these 4 patients underwent before the DeNovo NT graft implantation procedures and the defect sizes. The demographic background and early clinical outcome data of the 4 patients reported here are representative of the patient population $(N=25)$. Detailed data on all 25 patients will be presented in a subsequent expanded publication once all these patients have reached the 2-year postoperative follow-up milestone.

Figure 2 is an example of preoperative, 12-month, and 24-month postoperative MRI data for a defect treated with DeNovo NT particulated juvenile cartilage allograft tissue. It is apparent that good defect filling has been achieved for this patient at 24 months postoperative.

Figures 3, 4, and 5 graphically depict improvements in KOOS, IKDC outcome scores, and VAS pain scores over time for the 4 patients who have reached the 24-month postimplantation follow-up milestone. There were clear
Table 4. Defect Size/Location/Prior Surgeries

\begin{tabular}{|c|c|c|c|c|c|}
\hline Subject & $\begin{array}{c}\text { Size of } \\
\text { Lesion I, } \\
\mathrm{cm}^{2}\end{array}$ & $\begin{array}{c}\text { Size of } \\
\text { Lesion } 2 \\
\mathrm{~cm}^{2}\end{array}$ & $\begin{array}{c}\text { Location } \\
\text { of Lesion } \\
\text { । }\end{array}$ & $\begin{array}{l}\text { Location } \\
\text { of Lesion } \\
2\end{array}$ & Prior Surgeries \\
\hline I & 2.24 & & $\begin{array}{l}\text { Trochlear } \\
\text { groove }\end{array}$ & & \\
\hline 2 & 1.62 & & $\begin{array}{l}\text { Femoral } \\
\text { condyle } \\
\text { (medial) }\end{array}$ & & \\
\hline 3 & 2.85 & 4.62 & $\begin{array}{l}\text { Femoral } \\
\text { condyle } \\
\text { (lateral) }\end{array}$ & $\begin{array}{c}\text { Trochlear } \\
\text { groove }\end{array}$ & $\begin{array}{l}\text { Medial partial } \\
\text { meniscectomy }\end{array}$ \\
\hline 4 & 2.24 & & $\begin{array}{l}\text { Trochlear } \\
\text { groove }\end{array}$ & & $\begin{array}{l}\text { Cartilage } \\
\text { debridement; } \\
\text { medial partial } \\
\text { meniscectomy }\end{array}$ \\
\hline $\begin{array}{l}\text { Mean } \\
\text { (SD) }\end{array}$ & \multicolumn{2}{|c|}{2.71 (I.2) } & & & \\
\hline $\begin{array}{l}\text { Min, Max, } \\
N\end{array}$ & \multicolumn{2}{|c|}{ I. $62,4.62,5$} & & & \\
\hline
\end{tabular}

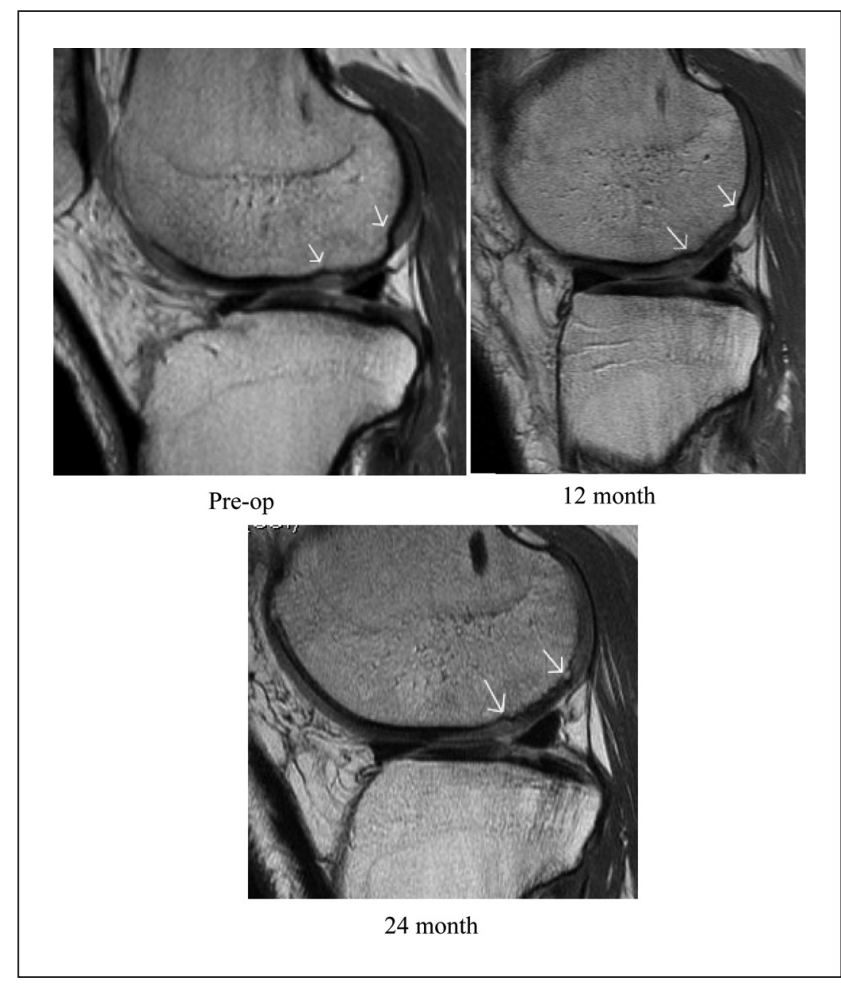

Figure 2. Note that the sagittal plane is not identical from I image to the next secondary to slight position differences of the knee in the MRI. The image plane that best demonstrated the lesion was chosen.

improvements in all scores across the 24-month follow-up period. Most of these improvements, especially in KOOS and VAS pain scores, were achieved at 12 months postsurgery and maintained throughout the 24-month follow-up period. 


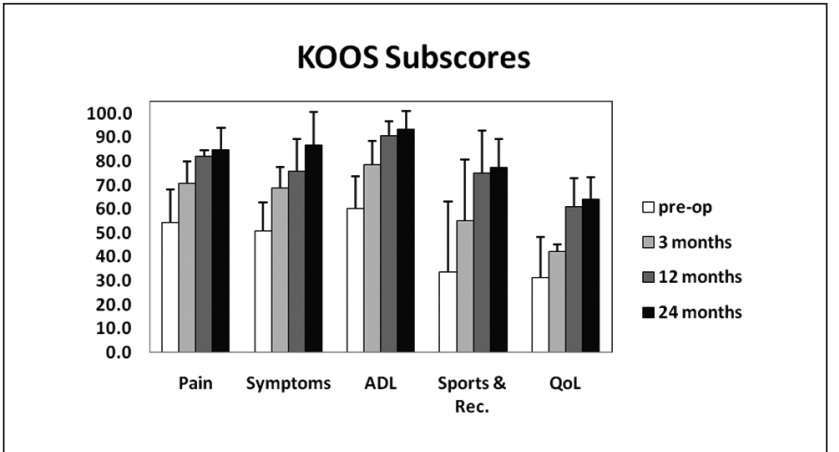

Figure 3. KOOS clinical outcome scores (Pain, Symptoms, ADL, Sports and Recreation, Quality of Life) for 4 patients who have reached the 24-month postimplantation milestone.

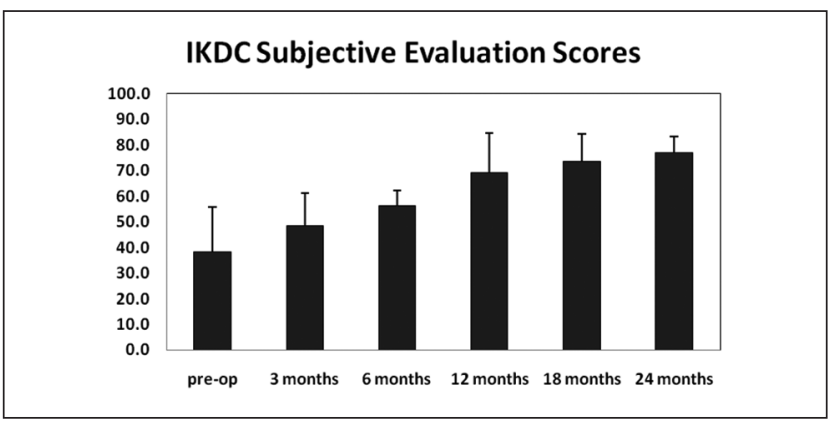

Figure 4. IKDC subjective clinical outcome measures for 4 patients who have reached the 24 -month postimplantation milestone.

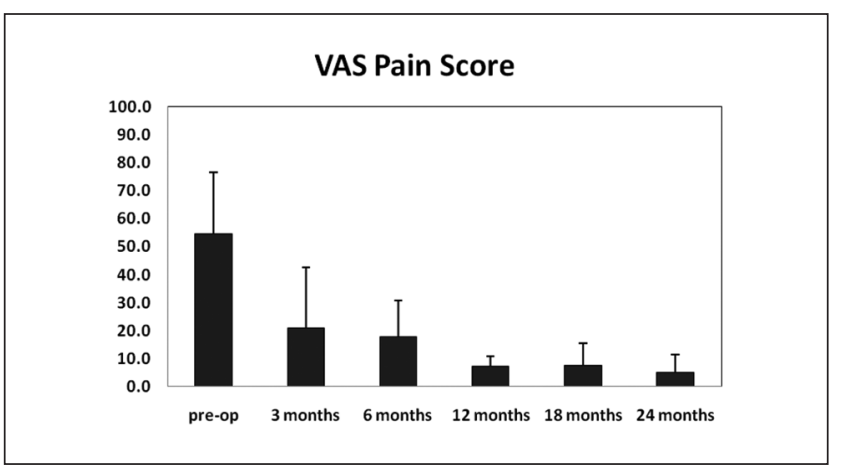

Figure 5. VAS pain measures for 4 patients who have reached the 24-month postimplantation milestone.

\section{Discussion}

\section{Key Findings of the Study}

Although the sample size is relatively small, the preliminary data from the first 4 patients indicate that this cartilage repair technique is capable of providing patients with greatly improved clinical outcomes and good defect filling.
This technique has several potential benefits. First, when repairing a chondral defect, there is no need to surgically create an osteochondral lesion (necessary for the fresh osteochondral allograft procedure), and consequently the biological challenge of transplant bone remodeling/ incorporation to host bone can be overcome. Second, there is no need to harvest autologous cartilage tissue as required for any autograft procedures (e.g., osteochondral autograft transplantation and autologous chondrocyte implantation). Third, the approach doesn't "burn bridges" because violation of the subchondral bone (necessary for marrow stimulation techniques such as microfracture) is not required. Marrow stimulation has been shown to compromise subsequent revision ACI surgeries. ${ }^{20}$ Fourth, the transplantation of particulated juvenile cartilage tissue allograft is a singlestage procedure, thus avoiding the need for 2 -stage procedures such as autologous chondrocyte implantation. The present study provides original clinical data on this promising cartilage repair technique. This is a prospective, singlearm study of a cohort of patients treated with particulated juvenile cartilage tissue allograft. It is not a randomized controlled trial. In addition, the present article only reports data from the initial 4 study subjects over follow-up periods up to 24 months. The preliminary data compare favorably to clinical data reported for other techniques. For instance, improvement of $30.5 \pm 11.3$ in the 2-year postoperative KOOS pain score from the preoperative baseline score compares favorably with results reported for microfracture and ACI. ${ }^{21}$ The IKDC subjective scores were also improved substantially from baseline $(38.9 \pm 12.6)$ at the 2-year postoperative evaluation. This compared favorably with results reported for ACI at $19.4 \pm 24.2$ and average values of 30 to 40 from other ACI studies. ${ }^{6,22,23}$ Subsequent data collected on more subjects and over longer follow-up periods will provide more evidence on the clinical performance of this technique.

\section{Risks Associated with Immunological Rejection/Disease Transmission}

Although osteochondral allograft transplantation is a clinically proven, efficacious cartilage restoration procedure, immune rejection, and disease transmission could pose risks to recipients. Fortunately, a significant amount of scientific data accumulated over the past decades elucidate concerns related to osteochondral transplants and provide support for the use of chondral transplants.

\section{Immunology}

- Clinical experience of more than 3 decades of fresh osteochondral allograft transplantation indicates that immune suppressants are not required for 
these procedures. Although elevated levels of relevant antibodies have been detected in the serum samples of recipients, there have been no reports of definite immunological rejection of transplanted osteochondral allografts with the exception of occasional lack of incorporation of the bony portion of the transplants. It is noteworthy, however, that Bugbee ${ }^{9}$ reported that (1) those patients with greater amounts of transplant tissue were more likely to be serum antibody positive and (2) antibody-positive patients had less optimal outcomes than did antibody-negative patients overall. The weak immune response is reported to arise from marrow elements retained within the osseous component of the osteochondral transplant. No immunological response to the cartilage component of osteochondral allografts has been reported.

- In vitro mixed lymphocyte reaction alloreactivity assays have shown that chondrocytes do not elicit an alloresponse as demonstrated by the lack of proliferation of human peripheral blood lymphocytes co-cultured with allogeneic human chondrocytes. This lack of an alloresponse was deemed to be attributable to the lack of chondrocyte expression of specific costimulatory molecules (e.g., CD80 and CD86) required for alloreactivity. Additionally, chondrocytes were recently reported to express cell surface proteins capable of suppressing active lymphocyte proliferation. ${ }^{24}$

- Articular cartilage has been shown to be immune privileged, ${ }^{25}$ partly because of the lack of vasculature and the dense extracellular matrix of this tissue.

Disease Transmission. To reduce the risk of disease transmission, the U.S. Food and Drug Administration (FDA) has established stringent requirements in "Donor Eligibility," 21 CFR Part 1271 subpart C, and "Guidance for Industry: Eligibility Determination for Donors of Human Cells, Tissues, and Cellular and Tissue-Based Products (HCT/Ps). Although the disease transmission risk is a concern for any allograft transplantation procedure, the rate of occurrence is extremely low. Extensive health and lifestyle screening of donors and family members is conducted. Additionally, standard screening tests are performed for each donor to ensure tissue safety. These tests are anti-human immunodeficiency virus (HIV) type 1 and 2, HIV nucleic acid test (NAT), hepatitis B surface antigen (HBsAg), anti-hepatitis B core antigen ( $\mathrm{HBcAb}$, total, IgG, and IgM), anti-hepatitis $\mathrm{C}$ virus (HCV), HCV NAT, anti-human T-lymphotropic virus (HTLV) type I and II, syphilis (RPR screening test, followed by a treponemal-specific assay if screening assay is positive). In addition to those tests required by the FDA, the following screening tests are performed: EBV (IgM)Epstein Barr virus and CMV (IgM)-cytomegalovirus and West Niles virus. Standard sterility testing was also conducted for each lot (donor) of DeNovo NT graft prior to tissue release.

Objectives and Limitations of MRI. The objectives of MRI performed at various time points in cartilage studies are to characterize the extent of changes to cartilage and subchondral bone qualitatively and/or quantitatively. For instance, the amount of defect filling and quality of repair tissue could be analyzed from MRI data. Any substantial subchondral bone changes could also been detected. These changes may be related or independent of actual repair procedures. One example is the subchondral bone edema often observed in patients who have received cartilage repair surgical treatments. Progression of subchondral bone edema should be monitored to understand the causes and consequences of these bony changes. However, there are limitations of MRI, such as difficulties in interpretation, measurement resolution, and cost.

Hypertrophy. Hypertrophy is often seen with $\mathrm{ACI},{ }^{26}$ with a subset of patients eventually requiring revision surgery to shave away the excessive amount of hypertrophic tissue. This tissue overgrowth has been found to be mainly due to the use of periosteal flap in ACI procedures, and replacement of the periosteal flap with a collagen membrane has largely resolved the issue. ${ }^{27}$ In the present study of chondral defect repair with particulated juvenile cartilage allograft, no periosteal flap is used and no revision was performed as the consequence of repair tissue hypertrophy.

\section{Conclusions}

Based on the preliminary clinical data obtained so far on the initial 4 patients who have reached the important milestone of 24 months postimplantation of DeNovo NT graft, patients experienced consistently encouraging improvement from baseline for parameters of pain and function as demonstrated by IKDC, KOOS, and VAS clinical outcome measures. The improvement observed in this preliminary report needs to be confirmed with the complete set of data for all 25 patients at 24 months of follow-up in a future report. In addition, MRI evaluations suggest good defect filling. There were no complications, and no evidence of graft rejection phenomena was reported. The outcomes suggest that particulated juvenile cartilage allograft is capable of filling chondral defects and greatly improving symptoms, given the understanding of the limitations of this study as a case series without a control arm. 


\section{Declaration of Conflicting Interests}

Dr. Jian Q. Yao is an employee of Zimmer, Inc. Dr. Jack Farr is a consultant of Zimmer, Inc. on biologics products/projects (but not a developer for this graft and he receives no royalties for this product).

\section{Funding}

Financial support was provided by Zimmer, Inc. through a research agreement [protocol ISTO-DeNovo NT 03-07-02].

\section{Acknowledgements}

The authors also would like to acknowledge the invaluable assistance of Vicki Snodgrass-Miller and Andrea Clifford at OrthoIndy, and Patrick Reischling and Nikesha Herrington at Zimmer, Inc.

\section{References}

1. Moseley JB, O’Malley K, Petersen NJ, Menke TJ, Brody BA, Kuykendall DH, et al. A controlled trial of arthroscopic surgery for osteoarthritis of the knee. N Engl J Med. 2002;347: 81-8.

2. Steadman JR, Rodkey WG, Singleton SB, Briggs KK. Microfracture technique for full-thickness chondral defects: technique and clinical results. Oper Tech Orthop. 1997;7:300-4.

3. Hangody L, Kish G, Kárpáti Z, Szerb I, Udvarhelyi I. Arthroscopic autogenous osteochondral mosaicplasty for the treatment of femoral condylar articular defects: a preliminary report. Knee Surg Sports Traumatol Arthrosc. 1997;5:262-7.

4. Gross AE, Shasha N, Aubin P. Long-term followup of the use of fresh osteochondral allografts for posttraumatic knee defects. Clin Orthop Relat Res. 2005;435:79-87.

5. Brittberg M, Lindahl A, Nilsson A, Ohlsson C, Isaksson O, Peterson L, et al. Treatment of deep cartilage defects in the knee with autologous chondrocyte transplantation. N Engl J Med. 1994;331:889-95.

6. Zaslav K, Cole B, Brewster R, DeBerardino T, Farr J, Fowler $\mathrm{P}$, et al. A prospective study of autologous chondrocyte implantation in patients with failed prior treatment for articular cartilage defect of the knee: results of the Study of the Treatment of Articular Repair (STAR) clinical trial. Am J Sports Med. 2009;37:42-55.

7. Farr J. DeNovo NT natural graft tissue. 8th World Congress of the International Cartilage Repair Society; 2009 May 24-25; Miami, FL.

8. Bentley G. Articular tissue grafts. Ann Rheum Dis. 1992; 51:292-6.

9. Bugbee WD. Fresh osteochondral allografts. J Knee Surg. 2002;15:191-5.

10. Stone KR, Walgenbach AW, Freyer A, Turek TJ, Speer DP. Articular cartilage paste grafting to full-thickness articular cartilage knee joint lesions: a 2- to 12-year follow-up. Arthroscopy. 2006;22:291-9.
11. Albrecht F, Roessner A, Zimmermann E. Closure of osteochondral lesions using chondral fragments and fibrin adhesive. Arch Orthop Trauma Surg. 1983;101:213-7.

12. Lu Y, Dhanaraj S, Wang Z, Bradley DM, Bowman SM, Cole BJ, et al. Minced cartilage without cell culture serves as an effective intraoperative cell source for cartilage repair. J Orthop Res. 2006;24:1261-70.

13. Frisbie DD, Lu Y, Colhoun HA, Kawcak CE, Binette F, McIlwraith CW. In vivo evaluation of a one step autologous cartilage resurfacing technique in a long term equine model. 51st Annual Meeting of the Orthopedic Research Society; 2005; Washington, DC. February 20-23, 2005

14. Cheung HS, Cottrell WH, Stephenson K, Nimni ME. In vitro collagen biosynthesis in healing and normal rabbit articular cartilage. J Bone Joint Surg Am. 1978;60:1076-81.

15. Namba RS, Meuli M, Sullivan KM, Le AX, Adzick NS. Spontaneous repair of superficial defects in articular cartilage in a fetal lamb model. J Bone Joint Surg Am. 1998; 80:4-10.

16. Stockwell RA. The interrelationship of cell density and cartilage thickness in mammalian articular cartilage. J Anat. 1971; 109:411-21.

17. Feder J, Adkisson HD, Kiser N, Hruska K, Cheung R, Grodzinsky A, et al. The promise of chondral repair using neocartilage. In: Tissue engineering in musculoskeletal clinical practice. Rosemont, IL: AAOS; 2004. p. 219-26.

18. Adkisson HD, Martin JA, Amendola RL, Milliman C, Mauch KA, et al. The potential of human allogeneic juvenile chondrocytes for restoration of articular cartilage. Am J Sports Med. 2010;38:1324-33.

19. Rodrigo J, Steadman J, Silliman J, Fulstone H. Improvement of full-thickness chondral defect healing in the human knee after debridement and microfracture using continuous passive motion. Am J Knee Surg. 1994;7:109-16.

20. Minas T, Gomoll AH, Rosenberger R, Royce RO, Bryant T. Increased failure rate of autologous chondrocyte implantation after previous treatment with marrow stimulation techniques. Am J Sports Med. 2009;37:902-8.

21. Saris DBF, Vanlauwe J, Victor J, Almqvist KF, Verdonk R, Bellemans $\mathrm{J}$, et al. Treatment of symptomatic cartilage defects of the knee: characterized chondrocyte implantation results in better clinical outcome at 36 months in a randomized trial compared with microfracture. Am J Sports Med. 2009; 37(Suppl 1):10S-19S.

22. Crawford DC, Heveran CM, Cannon WD, Foo LF, Potter HG. An autologous cartilage tissue implant NeoCart for treatment of grade III chondral injury to the distal femur: prospective clinical safety trial at 2 years. Am J Sports Med. 2009;37: 1334-43.

23. Kon E, Gobbi A, Filardo G, Delcogliano M, Zaffagnini S, Marcacci M, et al. Arthroscopic second-generation autologous chondrocyte implantation compared with microfracture 
for chondral lesions of the knee: prospective nonrandomized study at 5 years. Am J Sports Med. 2009;37:33-41.

24. Adkisson HD, Milliman C, Zhang X, Mauch K, Maziarz RT, Streeter PR, et al. Immune evasion by neocartilage-derived chondrocytes: implications for biologic repair of joint articular cartilage. Stem Cell Res. 2010;4:57-68.

25. Gibson T, Brian Davis W, Curran R. The long-term survival of cartilage homografts in man. Br J Plast Surg. 1959;11:177-87.
26. Kreuz PC, Steinwachs M, Erggelet C, Krause SJ, Ossendorf C, Maier D, et al. Classification of graft hypertrophy after autologous chondrocyte implantation of full-thickness chondral defects in the knee. Osteoarthritis Cartilage. 2007;15:1339-47.

27. Gomoll A, Probst C, Farr J, Cole B, Minas T. Use of a type I/III bilayer collagen membrane decreases reoperation rates for symptomatic hypertrophy after autologous chondrocyte implantation. Am J Sports Med. 2009;37:205-35. 